\title{
Genital Filariasis - A Recurred Case of Post Operated Hydrocele
}

\author{
Mahima Laxmikant Shete ${ }^{1}$, Anahita V. Bhesania Hodiwala²
}

1,2 Department of Microbiology, MGM Medical College and Hospital, Kamothe, Maharashtra, India.

\section{INTRODUCTION}

Lymphatic filariasis, commonly termed as elephantiasis has been recorded since ancient Indian, Chinese, Egyptian and Persian writings. ${ }^{1}$ It is caused by a slender thread like nematode called Wuchereria bancrofti belonging to super family filaroidea. ${ }^{2}$ It exists in two morphological forms: Adult form and larvae. The adult form has a predilection for intrascrotal lymphatic vessels in hosts; thus hydrocele is one of the most common manifestations of bancroftian filariasis. ${ }^{3}$ Individuals having circulating microfilaria are outwardly healthy but have the ability to transmit infection to others through mosquito bites (Culex quinquefasciatus) ${ }^{1}$ In developing countries like India, occurrence of genital filarial hydrocele is more common in people living in bancroftian endemic areas or in people who were previously operated for eversion of sac. We hereby report a case of hydrocele fluid with microfilaria- an incidental finding on microscopy.

Wuchereria bancroti a filarial nematode inhabiting the lymphatics and transmitted through vector - culex mosquito. We hereby present a case of recurrence of genital filariasis. The patient presented with swelling of the left scrotal region with pain of dragging type. On evaluation, eosinophilia was not seen. He had a history of right hydrocele 28 years back. The aspirated hydrocele on direct microscopic examination showed presence of actively motile microfilaria which was confirmed on Giemsa staining. Recurrence is more common in bancroftian filarial endemic areas.

\section{PRESENTATION OF CASE}

A 58-year-old male patient visited Surgery OPD. He had been observing a swelling on the left scrotal region since 8 to 10 years which was small initially and increased gradually. He came to the casualty when started to experience pain of dragging type which increased on walking and reduced on lying down. Patient had a history of surgery for hydrocele of right scrotum approx. 28 years back. The scar of the surgery was still present during admission. Swelling was present covering the whole left scrotum. The skin over the swelling appeared normal. There was no rise in temperature and no change with cough. Pulse was 88 / min. Blood pressure: 130 / 80 $\mathrm{mmHg}$ in right upper arm in supine position. Respiratory Rate: 18 / min. ECG was within normal Limits

\footnotetext{
Lab Investigations (Blood)

- TLC: 10,890

- $\mathrm{Hb}(\mathrm{g} \%): 16.8$

- PT: 11.10 .94

- LFT: Within Normal Limits

- RFT: Within Normal Limits

- HHH: Non- Reactive

- No eosinophilia observed
}

Corresponding Author: Ms. Mahima Laxmikant Shete, 75/502, Ekveera Prasad, L. T Road No. 5, M. G Road, Goregaon (West), Mumbai - 400104, Maharashtra, India. E-mail: shetemahima@gmail.com

DOI: $10.14260 / \mathrm{jemds} / 2021 / 419$

How to Cite This Article: Shete ML, Hodiwala AVB. Genital filariasis a recurred case of post operated hydrocele. J Evolution Med Dent Sci 2021;10(27): 2048-2050, DOI:

10.14260/jemds/2021/419

Submission 23-02-2021,

Peer Review 06-05-2021,

Acceptance 12-05-2021,

Published 05-07-2021.

Copyright (c) 2021 Mahima Laxmikant Shete et al. This is an open access article distributed under Creative Commons Attribution License [Attribution 4.0 International (CC BY 4.0)] 

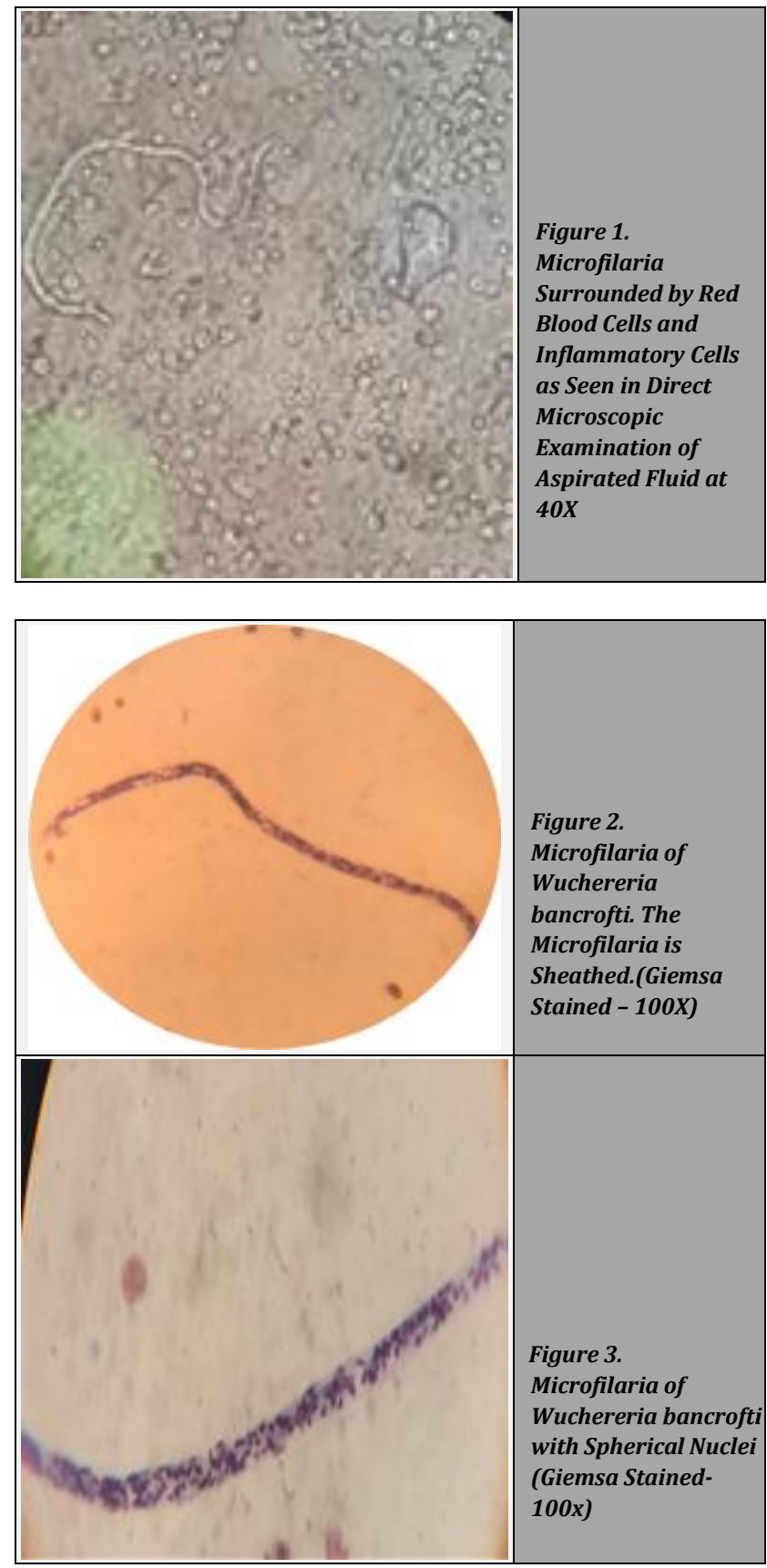

\section{DISCUSSION OF MANAGEMENT}

\section{Surgery}

- Eversion of left scrotal sac.

- Hydrocele fluid was sent to Microbiology lab for routine Culture sensitivity to rule out bacterial infection. However, on

- Direct microscopic examination of the aspirated fluid presence of actively motile microfilaria were observed. (FIG. 1). On trying to elicit history again, it was found that the patient originally belonged to Latur which is a bancroftian filariasis endemic zone. Smears of the sample were prepared and Giemsa staining done which confirmed presence of microfilaria (Figure $2 \& 3$ ).

\section{DISCUSSION}

Indian physician Sushruta was the first to describe elephantiasis. Microfilaria of Wuchereria bancrofti was first discovered by Demarquay in hydrocele fluid in the year $1863 .{ }^{4}$ Chronic hydrocele is the accumulation of fluid around the testis leading to an increase in the volume of the scrotal contents.

Depending on the volume of fluid, hydrocele can be disfiguring and even incapacitating. Chronic hydrocele has multiple aetiologies, but irrespective of the cause, surgery is the standard form of treatment, and this can be done using different surgical techniques. ${ }^{5}$ Genital bancroftian filariasis may manifest in several ways including hydrocoele, lymph varix, lymph scrotum, filarial penis or elephantiasis of the genitalia and chyluria.

Hydrocele accounts for $90 \%$ of the morbidity due to genital filariasis. Diagnosis of genital filariasis can be confirmed by direct demonstration of microfilaria in blood or aspirated fluid. The tools available for the detection of active infection in a microfilaremic patient are circulating filarial antigen (CFA) tests and the ultrasound with filarial dance sign (FDS).

The adult $W$. bancrofti may produce lesions by involving the lymphatics of the lower limbs, spermatic cord, epididymis, testis, retroperitoneum, and female breast. ${ }^{1}$ Its typical presentations are elephantiasis, funiculitis, and lymphadenitis.

Clinically filariasis can be of two major categories: Filariasis of skin and subcutaneous tissue and lymphatic filariasis. In the present case, microfilaria was seen in the lymphatics. The life cycle of Wuchereria bancrofti is found in two hosts - Man is the definitive host and Mosquito is the intermediate host. The adult resides in the lymphatics where gravid female releases a large number of microfilaria. ${ }^{1}$

A standardized clinical classification of filarial hydrocele has been proposed by Capuano and Capuano which is based on 4 criteria:

- $\quad$ Type- Unilateral versus bilateral

- $\quad$ Side (left / right)

- Scrotal enlargement- Rated from I to VI

- Grade of burial of penis- Rated from 0 to 4 .

\section{Grading Scale for Size of Scrotum}

- Stage I - The size of scrotum is less than that of a tennis ball

- Stage II - Size of scrotum becomes greater than that of the tennis ball up and down. It doesn't reach the halfway down the thigh.

- Stage III - Lower pole goes down to the mid-thigh and reaches the knee.

- $\quad$ Stage IV - The lower pole of the scrotum reaches the area between the upper pole of the patella and the lower pole of the knee.

- Stage V - The lower pole reaches the area between the lower edge of the knee and mid leg.

- Stage VI - The lower pole of the scrotum reaches the area between the mid leg and the ankle. 


\section{For Burial of Penis}

Burial of penis which often accompanies hydrocele, can be assessed on the patient lying down or standing. The burial can be classified according to its importance in 5 categories:

- Grade 0 - No apparent burial, the length of the penis is within normal limits.

- $\quad$ Grade 1 - Partial burial, the length of the visible part of the penis is greater than $2 \mathrm{~cm}$.

- $\quad$ Grade 2 - The length of the visible part of the penis is less than $2 \mathrm{~cm}$.

- $\quad$ Grade 3 - Total burial of the penis. Only the tip of the glans penis is visible.

- Grade 4 - Total burial of the penis. Causes micturition problems. ${ }^{6}$

The very significant difference in hydrocele rate recurrence observed between patients is two different surgical approaches- 1 . Complete excision and 2. Eversion with or without partial excision of sac. It may also signify that the pathogenesis of filarial hydrocele is complex.

In our case, the patient showed a recurrence of hydrocele in a different side of scrotal sac after 15 years. The microfilaria was detected in the aspirated hydrocele fluid which was reddish in colour.

\section{CONCLUSIONS}

The purpose of the case report is to emphasize the need of proper history taking especially with reference to the area where the patient has resided in the past 10 - 15 years because in India many states are endemic to filariasis. Also a high rate of suspicion will go a long way in coming to accurate diagnosis for the cause of chronic / recurrent hydrocele and deciding the right type of surgery needed for such cases. People should not ignore even a slight swelling or deformity and differential diagnosis should always be considered on observing such signs.

To prevent hydrocele recurrence, complete excision of the hydrocele sac should be done if possible.

Financial or other competing interests: None.

Disclosure forms provided by the authors are available with the full text of this article at jemds.com.

\section{REFERENCES}

[1] Sastry AS. Essentials of medical parasitology. Pondicherry: Jaypee Brothers Medical 2014: p. 265.

[2] Kanade US, Dantkale SS, Chaware SA, et al. Microfilaria in preauricular subcutaneous nodule on cytology: an unusual presentation. Journal of Evolution of Medical and Dental Sciences 2012;1(4):382-6.

[3] Potdar AV, Dubey K, Kalyanshetti A. Scrotal filariasis: post operated case of hydrocele. International Journal of Science and Research 2016;5(9):288-90.

[4] Sastry AS. Essentials of medical parasitology. $2^{\text {nd }}$ edn. Pondicherry: Elsevier 2019: p. 210.

[5] Noroes J, Dreyer G. A mechanism for chronic filarial hydrocele with implications for its surgical repair. PLoS Negl Trop Dis 2010;4(6):e695.

[6] Capuano GP, Capuano C. Surgical management of morbidity due to lymphatic filariasis: the usefulness of a standardized international clinical classification of hydroceles. Trop Biomed 2012;29(1):24-38. 